\title{
EVALUACIÓN DE INVERSIONES A LARGO PLAZO EN EL SECTOR FARMACÉUTICO ${ }^{1}$
}

\section{EVALUATION OF LONG-TERM INVESTMENTS IN THE PHARMACEUTICAL SECTOR}

\author{
Martínez Garcés, Josnel ${ }^{2}$ \\ Universidad del Zulia-Venezuela
}

RECIBIDO: Agosto 08 de 2015

ACEPTADO: Noviembre 24 de 2015

DOI

\begin{abstract}
RESUMEN
El presente artículo tiene como objetivo analizar los métodos de evaluación de las inversiones a largo plazo en activos fijos, tecnología y capacitación del recurso humano, utilizados por el sector farmacéutico del estado de Zulia (Venezuela). Metodológicamente, es de tipología descriptiva, con un diseño no experimental de campo y transversal. La población fue constituida por las siete (7) farmacias inscritas y activas en el Registro Municipal de la Alcaldía Bolivariana del municipio Jesús Enrique Lossada del Estado de Zulia al mes de mayo de 2015. Se utilizó el cuestionario estructurado como instrumento de recolección de datos aplicado a los gerentes y/o administradores, quienes fueron las unidades informantes; el mismo fue validado por cinco (5) expertos y mediante el nivel de confiabilidad, siendo el Alpha-Cronbach de 0,89. Los resultados demuestran que si bien pocas empresas acuden a los métodos tradicionales para evaluar las inversiones en propiedades, planta, equipos y tecnología, la mayoría aplica los destinados a evaluar inversiones en capacitación del talento humano. En conclusión, los métodos menos usados son aquellos que requieren la aplicación de fórmulas aritméticas (VPN, TIR, IR), evidenciando así que estas empresas carecen de personal calificado para usar e interpretar estos últimos. Se recomienda maximizar su uso para garantizar la salud financiera de estas organizaciones, así como aplicar los correctivos cuando la capacitación brindada al trabajador no produzca el efecto esperado.
\end{abstract}

Palabras clave: Activos fijos, inversión a largo plazo, métodos de evaluación, talento humano, tecnología.

\begin{abstract}
This article aims to analyze the methods of assessing long-term investments in fixed assets, technology and training of human resources used by the pharmaceutical sector of Zulia (Venezuela). Methodologically, the typology is descriptive, with a non-experimental field not a cross design. The chosen sample consisted of seven (7) registered and duly active pharmacies in the Municipal Register of the Bolivarian Mayor City of Losada - State of Zulia in May 2015. A well structured questionnaire was used as an instrument for data collection applied to managers and / or administrators who were reporting units. It was validated by five (5) experts and the level of reliability using the Cronbach Alpha was 0.89 . Results show that while only a few companies flock to traditional methods to evaluate investments in property, plant, equipment and technology, the majority applies them to assess the investment used for the training of human talent. In conclusion, the least used methods are those that require the application of arithmetic formulas (NPV, IRR, IR) thus showing that these companies lack qualified personnel for the use and interpretation of these methods. It is strongly recommended, in order to maximize its use, to ensure that these organizations are financially healthy, and at the same time proceed to apply the necessary correctives when the training provided to the worker does not produce the desired effect.
\end{abstract}

Keywords: Assessment methods, fixed assets, human talent, long term investment, technology.

Este artículo se puede referenciar

Martínez, J. (2016). Evaluación de inversiones a largo plazo en el sector farmacéutico. En Desarrollo Gerencial Revista de la Facultad de Ciencias Económicas Administrativas y Contables de la Universidad Simón Bolívar-Colombia, 8(1), 35-48.

1 Este artículo constituye un avance del proyecto de investigación (CH-0458-15) "Estrategias de inversión a largo plazo en sector farmacéutico del estado Zulia" registrado ante el Consejo de Desarrollo Científico y Humanístico (CONDES-LUZ) y adscrito al Centro de Estudios de la Empresa de la Universidad del Zulia (Venezuela).

2 Investigador adscrito al Centro de Estudios de la Empresa de la Facultad de Ciencias Económicas y Sociales - Universidad del Zulia (Venezuela). Docente catedrático de la Facultad de Ciencias Económicas, Administrativas y Contables. Correo: martinezjosnel@gmail.com 


\section{INTRODUCCIÓN}

En este siglo XXI es imprescindible la toma de decisiones gerenciales eficientes para el logro de metas y objetivos a fin de alcanzar el objetivo básico financiero: la obtención de ganancias. Esto va acompañado de la necesidad de satisfacer las exigencias de los consumidores a quienes las empresas ofrecen sus bienes y prestan sus servicios. Dicha toma de decisiones en la empresa es el producto de una ardua labor administrativo-financiera nacida en la fase inicial del proceso de planificación, definido por París (2005) como una toma de decisiones de lo que se pretende hacer. Sin embargo, estas decisiones se ven también condicionadas por factores internos (según la tipología de la empresa) y externos (según el entorno donde desarrolla sus actividades) que inciden en el desarrollo de las actividades empresariales (Bravo et al, 2010).

Como afirman Febles y Oreja (2008), internamente la empresa debe identificar cuáles son los recursos capaces de proporcionar una ventaja competitiva sustentable como elemento diferenciador, y externamente, hacer frente a las turbulencias y tendencias del entorno que definen los cambios organizacionales.

En el caso venezolano, estos factores han afectado de manera negativa el sano desarrollo de muchas empresas e industrias, llevando a muchas a su extinción. En relación al sector farmacéutico, la crisis generalizada en la producción de medicamentos, el desabastecimiento generalizado, las regulaciones y las restricciones en la obtención de divisas para la importación dificultan la toma de decisiones a quienes administran estas organizaciones. Esta idea es respaldada por Freddy Ceballos (2013), presidente de la Federación Farmacéutica Venezolana, quien afirma que las debilidades presentes en el sector durante el año 2010 se mantuvieron hasta el 2013, registrando más de un 50\% de fallos en la distribución de medicamentos.

Adicional a las debilidades mencionadas, Conde (2013) considera que la regulación de los precios, la falta de dotación de medicamentos por parte del Ministerio del Poder Popular para la Salud a los centros hospitalarios públicos y la política de medicamentos genéricos constituyen aspectos negativos que no han permitido sano crecimiento y desarrollo del sector. Esto ha obligado a recurrir constantemente a las importaciones como medio de obtención de los productos; pero las divisas son otorgadas de manera muy restrictiva, generando serias faltas de insumos y medicamentos en las farmacias.

A pesar de ello, para algunos propietarios de farmacias, el sector ha tenido un crecimiento en cuanto a la inversión a largo plazo, probablemente debido a la aplicación de estrategias eficientes (Koteich, 2014). Sin embargo, como indica Baca (2010), toda estrategia de inversión va necesariamente acompañada de su evaluación para determinar su rentabilidad económica. Esto da pie a la pregunta: ¿Cuáles son los métodos de evaluación de las inversiones utilizados en el sector farmacéutico del estado Zulia?, que a su vez fija el ob- 
jetivo de esta investigación: analizar los métodos de evaluación de las inversiones a largo plazo en activos fijos, tecnología y capacitación del recurso humano utilizados por el sector farmacéutico del estado Zulia.

\section{Estrategias de inversión a largo plazo}

Las estrategias gerenciales en el campo financiero pueden ser de tres tipos: 1) de inversión a corto y largo plazo; 2) de financiamiento a corto y largo plazo; y 3) para la gestión de tesorería (Van Horne y Wachowicz, 2010). Específicamente, las estrategias de inversión a largo plazo persiguen la adquisición de bienes activos y productos capaces de generar utilidad mediante la producción de otros bienes y servicios (Jiménez et al, 2003).

Bodie et al (2004) amplían el concepto al exponer que este tipo de estrategias se aplican generalmente durante el establecimiento de una empresa o para el mejoramiento de una ya establecida. Santandreu y Santandreu (2000) añaden que estas estrategias suponen la inmovilización de fondos durante un período mayor a un año, convirtiéndose así en decisiones de carácter estratégico. Algunas de ellas son la reposición o adquisición de activos fijos y la investigación y desarrollo.

Se debe tomar en cuenta que las inversiones a largo plazo no solo se relacionan con activos tangibles, sino también con activos inmateriales, que a pesar de no existir en términos físicos tienen un alto valor. Entre los más comunes están: los programas informáticos, las patentes, los conocimientos técnicos y los recursos humanos expertos (Garay y González, 2005), así como los derechos de autor, las marcas registradas y la plusvalía (Brigham y Houston, 2008).

Rivera (1993) señala al respecto que la necesidad de recursos humanos y el nivel de cualificación exigido a los mismos son ejemplos de las cosas en qué debe invertir la organización si desea establecer una posición competitiva en el largo plazo. En este sentido, Neilson (2008) propone un estudio previo de las competencias necesarias en los trabajadores, a fin de proporcionar la formación necesaria y evaluar la eficacia de las acciones tomadas. Esto debe acompañarse de estrategias encaminadas a que el personal sea consciente de la importancia y pertinencia de sus actividades para alcanzar los objetivos empresariales.

Yánez y Acevedo (2012) consideran este tipo de inversiones como altamente beneficiosas para la empresa, pues, incrementan la productividad de quienes reciben entrenamiento dentro de la organización. En tanto que para García et al (2012) el desarrollo del talento humano está claramente vinculado con la obtención de una ventaja competitiva sostenible y, por lo tanto, con el logro de mejores resultados económicos. 
Así, las estrategias de inversión se resumen en la evaluación, selección y ejecución de inversiones a largo plazo dentro de la empresa. Este proceso tiene una incidencia directa en decisiones como la de iniciar un nuevo proyecto, ampliar un área existente, adquirir activos de capital, reasignar recursos o llevar a cabo procesos de fusiones o adquisiciones (Useche, 2013).

Por esto, antes de que una organización invierta en activos debe considerar la importante relación de estos con el nivel de operaciones que realiza: poca inversión ahorca la operación, pero mucha inversión, la aplasta. En este mismo orden de ideas, para medir el desempeño de las decisiones de inversión tomadas en el negocio, se deben aplicar métodos de evaluación que contribuyan a la toma de decisiones gerenciales (Bravo et al, 2010). Estos métodos se aplican para comprobar la rentabilidad económica de los proyectos de inversión tomando en cuenta el valor del dinero en el tiempo (Baca, 2010), que según Emery et al (2000) incluye cuatro aspectos: el Valor Presente Neto, la Tasa Interna de Retorno, el Índice de Rentabilidad y el Período de Recuperación.

\section{MÉTODO}

A continuación se presenta el método utilizado en el desarrollo de esta investigación.

\section{Diseño}

En función del objetivo propuesto para este trabajo y considerando la tipología de investigación como el grado de profundidad con el cual se pretende abordar el fenómeno, el mismo se considera de tipo descriptivo. En relación al diseño desarrollado o la estrategia concebida para responder a las preguntas de investigación, la presente investigación puede considerarse con un diseño de campo y transversal, ya que no hay manipulación de la variable y el instrumento se aplicó in situ y en un único momento. Asimismo, se considera como una investigación no experimental, debido a que en ninguna etapa de la investigación se ha sometido la variable a condiciones específicas capaces de alterar su comportamiento.

\section{Participantes}

Respecto a la población o conjunto de elementos, seres o eventos sobre los cuales se desea obtener información, en la presente investigación se consideraron 11 farmacias inscritas en el Registro Municipal de la Alcaldía Bolivariana del municipio Jesús Enrique Lossada, del Estado Zulia (Venezuela) en mayo de 2015. Sin embargo, al momento de abordarlas solo siete (7) de ellas se encontraban operando activamente. Las mismas se muestran en el Cuadro 1. 
Cuadro 1. Distribución de la población

\begin{tabular}{|l|l|}
\hline $\mathbf{N}^{\mathbf{0}}$ & \multicolumn{1}{|c|}{ Nombre de la empresa } \\
\hline 01 & Farmacia Boulevard Francia, C.A. \\
\hline 02 & Farmacia Eduar, C.A. \\
\hline 03 & Farmacia Farmabien, C.A. \\
\hline 04 & Farmacia Las Amalias, C.A. \\
\hline 05 & Farmacia La Concepción, C.A. \\
\hline 06 & Farmacia Medifarmacia, C.A. \\
\hline 07 & Farmacia Virgen del Valle, C.A. \\
\hline
\end{tabular}

Fuente: Elaboración propia, a partir de información suministrada por el Registro Municipal de la Alcaldía Bolivariana del municipio Jesús Enrique Lossada del estado Zulia - Venezuela (Mayo 2015).

Las unidades informantes fueron los gerentes generales y/o administradores de cada una de las unidades. Para obtener la información no fue necesario aplicar técnicas de muestreo. Así, la población a estudiar se consideró un censo poblacional, pues es posible tocar cada uno de los elementos que la componen; además, la población es finita y totalmente accesible al investigador. El censo poblacional es la muestra en la cual entran todos los miembros de la población. Es el tipo de muestra más representativa.

\section{Instrumentos}

En la presente investigación se empleó la técnica de la observación, a través de instrumentos de recolección de datos como el cuestionario (Ver Anexo 1). El cuestionario fue validado por cinco (5) expertos en gerencia financiera, quienes se encargaron de constatar la pertinencia de los ítems con la variable, dimensiones e indicadores establecidos. La confiabilidad fue medida calculando el coeficiente de Alpha-Cronbach, que arrojó un resultado de 0,89. Esto sugiere un alto grado de confianza en el instrumento de recolección de datos.

\section{Procedimiento}

Inicialmente, se detectó el problema de estudio a fin de definir los objetivos que permitieran solucionar dicha problemática. Luego se seleccionaron las teorías para sustentar el estudio con base en la variable abordada. Ya una vez establecida la metodología, se diseñó el instrumento de recolección de datos para su posterior validación y análisis de confiabilidad. Tras aplicar el instrumento en la población objeto de estudio, se tabularon y analizaron los datos para finalmente generar los resultados y conclusiones presentadas en este artículo. 


\section{RESULTADOS}

Para Van Horne y Wachowicz (2010), los métodos de evaluación de las estrategias de inversión son cuatro: a) el periodo de recuperación, b) el valor presente neto, c) el índice de rentabilidad y d) la tasa interna de retorno. Estos representan métodos de evaluación previa a la inversión y se aplican en los casos de propiedades, planta y equipos, así como en la adquisición y mejora de tecnología, ya que todos estos casos pueden ser claramente cuantificables. Baca (2010) considera muy útil el uso de estos métodos de evaluación, pues toman en cuenta el cambio real del valor del dinero a través del tiempo. Según León (2012), el uso de uno o varios de estos procura optimizar la inversión dentro de los proyectos financieros.

En la Tabla 1 se presentan los métodos de evaluación de las inversiones a largo plazo en propiedades, planta y equipos, y adquisición y/o mejora de tecnología utilizados en el sector farmacéutico del estado Zulia.

Tabla 1. Métodos de evaluación de las inversiones en propiedades, planta y equipos en el sector farmacéutico del estado Zulia

\begin{tabular}{|l|c|c|c|c|c|c||}
\hline \multirow{2}{*}{ Método de evaluación utilizado } & \multicolumn{2}{|c|}{$\begin{array}{c}\text { No de empresas } \\
\text { que aplican el } \\
\text { método }\end{array}$} & $\begin{array}{c}\text { No de empresas } \\
\text { que no aplican el } \\
\text { método }\end{array}$ & \multicolumn{3}{|c|}{ Total } \\
\cline { 2 - 8 } & Fa & Fr & Fa & Fr & Fa & Fr \\
\hline Período de recuperación & 5 & 71,43 & 2 & 28,57 & 7 & 100,00 \\
\hline Valor presente neto & 2 & 28,57 & 5 & 71,43 & 7 & 100,00 \\
\hline Índice de rentabilidad & 3 & 42,86 & 4 & 57,14 & 7 & 100,00 \\
\hline Tasa interna de retorno & 3 & 42,86 & 4 & 57,14 & 7 & 100,00 \\
\hline
\end{tabular}

Fa: Frecuencia absoluta; Fr: Frecuencia relativa

Fuente: Elaboración propia (2015), a partir de los resultados obtenidos de un cuestionario aplicado durante el mes de junio de 2015 a gerentes y/o administradores de las siete farmacias activas y registradas en el Registro Municipal de la Alcaldía Bolivariana de Jesús Enrique Lossada, estado Zulia (Venezuela).

Sobre el período de recuperación, solo $71,43 \%$ de los encuestados conoce este método, quedando claro que más de la mitad establece lapsos determinados para la recuperación de la inversión, mientras el resto no lo hace. Un segundo método de evaluación es el valor presente neto. En este caso, 71,43\% de los encuestados lo desconoce; las pocas empresas que lo utilizan para evaluar sus inversiones establecen un monto esperado como Valor Presente Neto para efectuar una inversión.

Con respecto al índice de rentabilidad, $57,14 \%$ de los encuestados manifestó no conocer el método. Las 
empresas que sí lo utilizan conocen la relación costo actual-beneficio futuro, aplicándolo para evaluar sus inversiones. Un último método para evaluar la inversión en propiedades, planta y equipos y la adquisición o mejora de tecnológica es la tasa interna de retorno. Entre los encuestados, se determinó que más de la mitad, es decir un $57,14 \%$ de las empresas, no calcula un porcentaje mínimo de retorno para determinar la ejecución de la inversión. Solo 42,86\% conoce y utiliza este método.

Estos resultados demuestran que para la evaluación de las inversiones a largo plazo en propiedades, planta y equipos, y adquisición y/o mejora de tecnológica en el sector farmacéutico del estado Zulia, el principal método utilizado es el período de recuperación, seguido en menor frecuencia por el índice de rentabilidad y la tasa interna de retorno.

En otro orden de ideas, en el caso de las inversiones en capacitación y desarrollo del recurso humano es necesario acudir a métodos de evaluación más cualitativos y los resultados del rendimiento de estas se evidencian en el largo plazo. Para las inversiones en propiedades, planta y equipos, así como para la tecnología la evaluación se hace antes de realizar la inversión; pero en el caso de la formación del talento humano, esta se hace en forma posterior a la aplicación de la estrategia con el objetivo de medir su impacto y alcance.

En la era moderna, resulta indispensable que los programas de capacitación y desarrollo evidencien su impacto positivo dentro de las organizaciones (Prieto et al, 2011). Para ello, de acuerdo con Mondy y Noe (1997), la evaluación del desarrollo del recurso humano se logra a través de a) las opiniones de los participantes, b) el alcance del aprendizaje y c) el cambio del comportamiento.

En la Tabla 2, se muestran los métodos de evaluación de las inversiones a largo plazo en capacitación y desarrollo del talento humano utilizados en el sector farmacéutico del estado Zulia.

Tabla 2. Métodos de evaluación de las inversiones en capacitación y desarrollo del talento humano en el sector farmacéutico del estado Zulia

\begin{tabular}{|l|c|c|c|c|c|c|}
\hline \multirow{2}{*}{ Método de evaluación utilizado } & \multicolumn{2}{|c|}{$\begin{array}{c}N^{0} \text { de empresas } \\
\text { que aplican el } \\
\text { método }\end{array}$} & $\begin{array}{c}\mathbf{N}^{0} \text { de empresas } \\
\text { que no aplican el } \\
\text { método }\end{array}$ & \multicolumn{3}{|c|}{ Total } \\
\cline { 2 - 8 } & Fa & Fr & Fa & Fr & Fa & Fr \\
\hline Opiniones de los participantes & 7 & 100,00 & 0 & 0,00 & 7 & 100,00 \\
\hline Alcance de los aprendizajes & 6 & 85,71 & 1 & 14,29 & 7 & 100,00 \\
\hline Cambios en el comportamiento & 6 & 85,71 & 1 & 14,29 & 7 & 100,00 \\
\hline
\end{tabular}

Fa: Frecuencia absoluta; Fr: Frecuencia relativa

Fuente: Elaboración propia (2015), a partir de los resultados obtenidos de un cuestionario aplicado durante el mes de junio de 2015 a gerentes y/o administradores de las siete farmacias activas y registradas en el Registro Municipal de la Alcaldía Bolivariana de Jesús Enrique Lossada, estado Zulia (Venezuela). 
Cuando se indagó si las empresas del sector farmacéutico en el estado Zulia consideraban importante la opinión de los participantes, un 100\% manifestó que sí. Sin embargo, solo 85,71\% de los gerentes admitió consultar con sus colaboradores la temática sobre la cual deseaban recibir formación. Al momento de investigar si tras la actividad de capacitación y desarrollo se indagan las percepciones de los empleados acerca de la misma, la mayoría manifestó aplicar métodos de evaluación posteriores a la actividad de capacitación y desarrollo.

Sobre la evaluación mediante el alcance de los aprendizajes se detectó que un 85,71\% de las empresas analizadas aplica esta evaluación, es decir, la mayoría. Asimismo, se conoció que los gerentes de estas empresas aplican pruebas posteriores para medir el nivel de aprendizaje y comparan los conocimientos del empleado antes y después de la capacitación.

Un último método para evaluar las inversiones en capacitación y desarrollo del recurso humano es el cambio en el comportamiento. En este sentido, $85,71 \%$ de las empresas encuestadas hace un seguimiento del comportamiento del trabajador en su puesto de trabajo después de los programas de formación. No obstante, pocas de estas empresas toman medidas posteriores cuando la formación brindada no logra el cambio de comportamiento esperado. Los gerentes entrevistados que lo hacen así manifiestan que estas medidas se basan en reforzar la capacitación brindada.

Para todos los encuestados, la inversión en formación permite al empleado modificar también sus patrones de conducta personal, influyendo directamente en su actitud hacia el trabajo. Según los gerentes de estas empresas, los cambios en el comportamiento permiten vincular la estabilidad emocional del trabajador con su rendimiento laboral.

Todos estos resultados demuestran que para la evaluación de las inversiones a largo plazo en capacitación y desarrollo del talento humano en el sector farmacéutico del estado Zulia, el principal método utilizado consiste en conocer las opiniones de los participantes. Sin embargo, también son altamente usados métodos para medir los alcances de los aprendizajes y hacer seguimiento a los cambios en el comportamiento.

\section{DISCUSIÓN}

El periodo de recuperación de una inversión representa el número de años requeridos para recuperar la inversión de efectivo inicial con base en los flujos de efectivo esperados. Si el período de recuperación calculado es menor que el período de recuperación máximo aceptable, la propuesta se acepta; de lo contrario se rechaza (Van Horne y Wachowicz, 2010). Sobre el uso de este método de evaluación, los resultados 
demuestran que más de la mitad de las empresas estudiadas lo conoce y utiliza al momento de tomar una decisión de inversión.

Así se comprueban los planteamientos de Gitman (2007), para quien este método de evaluación es el más popular debido a su simplicidad computacional y atractivo intuitivo. También lo considera atractivo porque toma en cuenta los flujos de efectivo más allá de las utilidades contables. Por otro lado, las desventajas del período de recuperación son que su valor adecuado es solo un número determinado de manera subjetiva y no es capaz de reconocer los flujos de efectivo ocurridos después de la recuperación hasta alcanzar el punto de equilibrio (es decir, igualar la inversión inicial).

El valor presente neto representa, por su parte, la diferencia entre el costo de una inversión y lo que vale, es decir, su valor en el mercado (Emery et al, 2000). Como el valor presente neto considera el valor temporal del dinero se concibe como una técnica del presupuesto de capital compleja donde se descuentan los flujos de efectivo de la empresa a una tasa específica. Según Van Horne y Wachowicz (2010), el valor presente neto de un proyecto de inversión debe ser igual a cero o más, a fin de ser aceptado; en caso contrario, se rechaza. Para Vecino et al (2015), el VPN es uno de los métodos de evaluación más utilizado por los directivos en las empresas, pues, tiene la ventaja de mostrar el valor que tiene el dinero a través del tiempo.

Sin embargo, los resultados obtenidos en la presente investigación no respaldan este antecedente teórico. En efecto, para el caso de las empresas del sector farmacéutico del estado Zulia, el Valor Presente Neto es un método poco utilizado, ya que, como sostienen Martínez y Aizenstat (2006) carece de flexibilidad. Más bien se enfoca en determinar un valor basado en proyecciones de un flujo de caja a una tasa de descuento determinada, ignorando así otros factores externos que pudiesen dar viabilidad a la inversión.

El índice de rentabilidad representa la razón del valor presente de los futuros flujos netos de efectivo de un proyecto con el flujo de salida de efectivo inicial del proyecto (Van Horne y Wachowicz, 2010). Moyer et al (2008) precisan que con este método se establece el rendimiento del valor presente neto de cada unidad monetaria de la inversión inicial; también consideran como criterio de aceptación que este arroje un resultado mayor o igual a 1. En este sentido, alrededor de la mitad de las empresas estudiadas utiliza este método y conoce la relación costo actual-beneficio futuro, aplicándolo para evaluar sus inversiones.

Un último método para evaluar la inversión en propiedades, planta y equipos y la adquisición o mejora de tecnología es la tasa interna de retorno (TIR), en otras palabras, el cálculo de una tasa de interés capaz de igualar los flujos de salida de efectivo-costo de una inversión con sus subsecuentes flujos de entrada de efectivo (Block y Hirt, 2005). El criterio de aceptación usado para este método consiste en comparar la tasa 
interna de retorno con la tasa de rendimiento mínima aceptable (Van Horne y Wachowicz, 2010). Lumby y Jones (2003) hacen énfasis en la importancia de este método, debido a que, como el VPN, considera el valor del dinero en el tiempo; sin embargo, en lugar de dar el valor agregado en términos monetarios, muestra el rendimiento en cifras porcentuales.

Los planteamientos citados en el párrafo anterior no se validan frente a las empresas encuestadas, pues, menos de la mitad hace uso de él. Como indican Ochoa y Mora (2014), el hecho de que la TIR muestre un rendimiento porcentual representa una limitante adicional a su cálculo, que es capaz de arrojar más de un valor cuando se tiene un flujo de caja no constante, pero que también es confuso de entender por ser un procedimiento aritmético.

El poco uso de estos tres últimos métodos de evaluación de las inversiones entre las farmacias del municipio Jesús Enrique Lossada demuestra la veracidad de los planteamientos de Gitman (2007), quien los considera complejos por requerir el conocimiento de técnicas presupuestarias muy específicas. Por otro lado, es evidente que estas empresas carecen de personal calificado para la aplicación, análisis e interpretación de dichos métodos.

Con pocas palabras, en el caso de la evaluación de las inversiones a largo plazo en propiedades, planta y equipos, y adquisición y/o mejora tecnológica en el sector farmacéutico del estado Zulia, estos resultados muestran que el principal método utilizado es el período de recuperación, seguido por el índice de rentabilidad y la tasa interna de retorno.

La evaluación de la inversión en programas de recursos humanos mediante la solicitud de opiniones de los participantes es un recurso de bajo costo, con el que se puede obtener una respuesta inmediata y sugerencias para el mejoramiento. Sin embargo, este método de evaluación presenta un problema: se basa en opiniones y no en hechos. En realidad, el capacitado pudiera no haber aprendido nada aun cuando crea haber aprendido (Pérez, 2006). En este sentido, todas las empresas encuestadas manifestaron tomar en cuenta las opiniones de sus trabajadores al momento de brindar una actividad de capacitación y/o actualización. La mayoría de las empresas estudiadas indaga sobre las percepciones del trabajador acerca de la misma; no obstante, la percepción de los participantes sobre el valor de dichos programas de capacitación y desarrollo puede ser útil pero debe ser interpretada con precaución (Robles, 2008).

En cuanto a la evaluación mediante el alcance de los aprendizajes, Fajardo et al (2010) la entienden como la administración de pruebas para determinar lo que han aprendido los participantes en un programa de capacitación y desarrollo del recurso humano. Se pueden diseñar grupos de control antes y después de la prueba. Cabe anotar que la mayoría de las empresas estudiadas conoce y aplica este tipo de evaluación. 
En su aplicación, se suele usar la misma prueba antes y después de la capacitación, estableciendo un grupo de control que no recibe capacitación y un grupo experimental que sí la recibe. Este método permite atribuir las diferencias de resultados de la prueba anterior y la prueba posterior entre los grupos con relación a la capacitación recibida (Márquez y Díaz, 2005). La mayoría de las empresas del sector farmacéutico del estado Zulia aplican pruebas posteriores a los beneficiados del programa de capacitación, a fin de medir sus alcances.

Un último método para evaluar las inversiones en capacitación y desarrollo del recurso humano es el cambio en el comportamiento, es decir, la aplicación de pruebas para reconocer con bastante precisión lo que se ha aprendido y saber si ha generado los cambios esperados en la conducta, especialmente en lo relacionado con la motivación del empleado (Hernández et al, 2009). La mayor parte de las empresas entrevistadas hace un seguimiento del comportamiento del trabajador en su puesto de trabajo después de los programas de formación, pero pocas toman medidas posteriores cuando la formación brindada no ha posibilitado el cambio de comportamiento esperado. Los gerentes entrevistados manifiestan que estas medidas se basan en reforzar la capacitación brindada.

Para todos los gerentes entrevistados, la inversión en formación permite al empleado modificar sus patrones de conducta personal, influyendo directamente en su actitud hacia el trabajo. Los cambios en el comportamiento permiten vincular la estabilidad emocional del trabajador con su rendimiento laboral en la empresa. Esto soporta lo afirmado por Alonso et al (2010), para quienes es imprescindible medir estos cambios, pues con la capacitación se espera una mejora de las actitudes del trabajador en el desarrollo de sus actividades, así como elevar su desempeño dentro de la organización.

Nuestros resultados demuestran que el principal método utilizado para evaluar las inversiones a largo plazo en capacitación y desarrollo del talento humano en el sector farmacéutico del estado Zulia consiste en conocer las opiniones de los participantes. Sin embargo, la medición de los alcances de los aprendizajes y hacer seguimiento a los cambios en el comportamiento también son altamente usados.

En general, de esta investigación también se concluye que la mayoría de las empresas encuestadas conoce el método Periodo de recuperación, y lo utilizan en la misma proporción para establecer lapsos mínimos de recuperación de inversión. En cambio, pocas de estas empresas conocen y utilizan el Valor Presente Neto (VPN), para establecer un monto esperado de VPN al momento de tomar decisiones.

Sobre el Índice de rentabilidad quedó claro que menos de la mitad de las compañías entrevistadas lo utiliza, relacionando el costo actual-beneficio futuro para evaluar la inversión. Igual ocurre con la Tasa interna de retorno, ya que menos de la mitad de los entrevistados sabe cómo se calcula y lo aplican. 
Estos cuatro métodos son los utilizados para evaluar las inversiones en propiedades, planta y equipos así como la tecnología, y se aplican antes de efectuar la inversión. De modo que las empresas estudiadas necesitan mejorar sus prácticas evaluativas sobre las inversiones. Esto les permitirá determinar si obtendrán los rendimientos esperados en el futuro.

En el caso de la inversión en capacitación y desarrollo del talento humano, se utilizan métodos posteriores para medir su rentabilidad y eficacia. Son básicamente tres: opiniones de los participantes, alcance de los aprendizajes y cambios en el comportamiento. En relación a la opinión de los participantes, todas las empresas encuestadas consideran importante la opinión de sus empleados. Concretamente, la mayoría consulta con ellos la temática sobre la cual desean recibir formación y aplican evaluaciones posteriores.

Sobre el alcance de los aprendizajes, se comprobó que la mayoría de las empresas entrevistadas siempre aplica este método, que compara el conocimiento de los trabajadores antes y después de recibir la formación. Lo mismo ocurre con el método de Cambios en el comportamiento: la mayoría de estas empresas hace seguimiento al trabajador en su puesto de trabajo una vez recibida la formación, aun cuando pocas aplican correctivos en caso de no obtener el efecto esperado. Todas las empresas en cuestión consideran que este tipo de inversión no solo es beneficioso para la empresa, sino para quien la recibe, pues permite modificar sus patrones de conducta, armonizando su vida personal con su entorno laboral.

En función de todo lo expuesto, se sugiere maximizar el uso de los métodos de evaluación tradicionales de la inversión en propiedades, planta y equipos, así como para la tecnología, pues la realidad demuestra que su aplicación resulta altamente positiva para la salud de las organizaciones. Por otro lado, se sugiere aplicar acciones correctivas cuando la capacitación brindada no produzca el efecto esperado tras aplicar las pruebas de cambios en el comportamiento. Así se evalúa eficazmente la inversión en capacitación y desarrollo del recurso humano.

\section{REFERENCIAS}

Alonso, P.; Sarmenteros, I.; Zamora, M. y Arreche, L. (2010). El impacto de la capacitación, algunas consideraciones a partir de una experiencia de su evaluación en un hotel todo incluido. Revista Avanzada Cientifica Vol. 13 No 3 pp. 36-47. Ministerio de Ciencia, Tecnología y Medio Ambiente. Cuba.

Baca, G. (2010). Evaluación de proyectos. México: Editorial McGraw Hill.

Block, S. y Hirt, G. (2005). Administración financiera. México: Editorial McGraw Hill.

Bodie, Z.; Kane, A. y Marcus, Al. (2004). Principios de inversión. Madrid: Editorial McGraw-Hill.

Bravo, M.; Lambretón, V. y Márquez, H. (2010). Introducción a las finanzas. México: Editorial Pearson Prentice Hall. 
Brigham, E. y Houston, J. (2008). Fundamentos de administración financiera. México: Ediciones Thompson.

Ceballos, F. (2013). Crisis en el sector farmacéutico se extenderá hasta el 2014. Disponible en: http:// elimpulso.com/articulo/crisis-en-el-sector-farmaceutico-se-extendera-hasta-el-2014. Consultado 03/04/2014.

Conde, D. (2013). La Federación de farmacias venezolana denuncia la falta de medicamentos. Disponible en: http://elimpulso.com/articulo/federacion-farmaceutica-venezolana-desabastecimiento-de-medicamentos-podria-superar-el-de-alimentos. Consultado 12/05/2014

Emery, D.; Finnerty, J. y Stowe, J. (2000). Fundamentos de administración financiera. México: Editorial Pearson Educación.

Fajardo, L., Hernández, E. y Molina, A. (2010). Consideraciones acerca de una metodología para evaluar el impacto de la capacitación en la ESIB. Revista Ingeniería Industrial Vol. 31 No 1 pp. 1-7. Instituto Superior Politécnico José Antonio Echeverría. Cuba.

Febles, J. y Oreja J. (2008). Factores externos e internos determinantes de la orientación de la cultura estratégica de las empresas. Investigaciones Europeas de Dirección y Economía de la Empresa Vol. $14 \mathrm{~N}^{\mathrm{o}}$ 1 pp. 13-31. Madrid: Academia Europea de Dirección y Economía de la Empresa.

Francés, A. (2001). Estrategias y planes para la empresa en el cuadro de mando integral. Editorial Pearson Educación. México.

Garay, U. y González, M. (2005). Fundamentos de finanzas con aplicación al mercado venezolano. Ediciones IESA. Venezuela.

García, L.; García, J. y Rodríguez, A. (2012). Impacto de la inversión en capital humano sobre el valor empresarial. Revista Latinoamericana de Administración $\mathrm{N}^{\circ} 51$ pp. 15-26. Consejo Latinoamericano de Escuelas de Administración. Colombia.

Gitman, L. (2007). Principios de administración financiera. México: Editorial Pearson Educación. Hernández, J.; Chumaceiro, A. y Atencio, E. (2009). Calidad de servicio y recurso humano: caso estudio tienda por departamentos. Revista Venezolana de Gerencia Año 14 No 47 pp. 457-470. Universidad del Zulia. Venezuela.

Jiménez, J.; Pérez, C. y De la Torre, A. (2003). Gestión financiera de la empresa. Madrid: Ediciones Pirámide.

Koteich, K. Gerente de Farmacia Medifarmacia, C.A.; La Concepción (Edo. Zulia - Venezuela), 04 de julio de 2014.

León, G. (2012). Análisis comparativo de los métodos tradicionales de valoración aplicado a la simulación de un proyecto de inversión. Revista Dimensión Empresarial Vol. 10 N 1 pp. 16-21. Universidad Autónoma del Caribe. Colombia.

Lumby. S. y Jones, C. (2003). Corporate finance theory and practice.Editorial Thompson. Inglaterra. 
Márquez, J. y Díaz, J. (2005). Formación del recurso humano por competencias. Sapiens Revista Universitaria de Investigación pp. 85-105. Universidad Pedagógica Experimental Libertador. Venezuela.

Martínez, F. y Aizenstat, A. (2006). Opciones reales, valuación financiera de proyectos y estrategias de negocios: aplicaciones al caso mexicano. Revista El trimestre económico Año LXXIII No 290 pp. 363-405. Fondo de Cultura Económica. Colombia.

Mondy, W. y Noe, R. (1997). Administración de recursos humanos. México: Editorial Pearson Educación. Moyer, Ch., Mcguigan, J. y Kretlow, W. (2008). Administración financiera contemporánea. México: Editorial Thomson.

Neilson, J. (2008). La realidad local de la evaluación de la capacitación. Revista Gestión de las Personas y Tecnología $\mathrm{N}^{\mathrm{o}} 1$ pp. 32-37. Universidad de Santiago de Chile. Chile.

Ochoa, J. y Mora, A. (2014). Prácticas de presupuesto de capital: evaluación empírica en un grupo de empresas del sector de la construcción en Colombia. Revista Ecos de Economía Vol. 18 No 39 pp. 143163. Universidad EAFIT. Colombia.

París, F. (2005). La planificación estratégica. Madrid: Editorial Paidotribo.

Pérez, D. (2006). El adiestramiento del recurso humano, herramienta de competitividad en la PYME. Revista Multiciencias Vol. 6 N² 2 pp. 148-153. Universidad del Zulia. Venezuela.

Prieto, R.; Villasmil, M. y Urdaneta, L. (2011). Gestión humana en organizaciones postmodernas. Base fundamental hacia la excelencia organizacional. Revista TELOS Vol. 8 Edición 2 pp. 1-12. Universidad Rafael Belloso Chacín. Venezuela.

Rivera, O. (1993). La interrelación entre estrategia y resultados económico-financieros. Revista Estudios Empresariales $\mathrm{N}^{\circ} 83$ pp. 35-48. Universidad DEUSTO. España.

Robles, N. (2008). Metodología para la evaluación de la eficacia y eficiencia en la capacitación del recurso humano. Revista Tecnología en marcha Vol. 21 N$^{\circ} 4$ pp. 51-59. Editorial Tecnológica de Costa Rica. Costa Rica.

Santandreu, E. y Santandreu, P. (2000). Manual de Finanzas. Madrid: Editorial Gestión.

Useche, A. (2013). Exceso de confianza y optimismo en las decisiones de presupuesto de capital: las finanzas corporativas desde un enfoque centrado en el comportamiento. Revista Universidad y Empresa Vol. $16 \mathrm{~N}^{\mathrm{o}} 26$ pp. 93-114. Universidad del Rosario. Colombia.

Van Horne, J. y Wachowicz, J. (2010). Administración financiera. México: Editorial McGraw Hill.

Vecino, C., Rojas, S. y Muñoz, Y. (2015). Prácticas de evaluación financiera de inversiones en Colombia. Revista Estudios Gerenciales Vol. 31 No 134 pp. 41-49. Universidad ICESI. Colombia.

Yánez, M. y Acevedo, K. (2012). Inversión en entrenamiento en el empleo: una revisión de la literatura. Revista Semestre Económico Vol. 15 No 31 pp. 153-172. Colombia. 